\title{
Fluorescence Resonance Energy Transfer Enhanced Luminescence (FRETEL) of Quantum Dyes ${ }^{\circledR}$
}

\author{
R. C. Leif ${ }^{* a}$, M. C. Becker ${ }^{\mathrm{b}}$, A. Bromm Jr. ${ }^{\mathrm{c}}$, L. M. Vallarino ${ }^{\mathrm{c}}$, and S. Yang ${ }^{\mathrm{a}}$ \\ ${ }^{a}$ Newport Instruments; ${ }^{b}$ Phoenix Flow Systems; ${ }^{c}$ Dept. of Chemistry, Virginia Commonwealth \\ University
}

\begin{abstract}
Methods for increasing the luminescence intensity of lanthanide macrocycles, Quantum Dyes ${ }^{\circledR}$, by the Fluorescence Resonance Energy Transfer Enhanced Luminescence (FRETEL) effect in the solid state have been developed. A homogeneous solution containing the europium or terbium Quantum Dye and an excess of selected energy transfer species is evaporated to dryness, resulting in a thin film that surrounds and embeds the Quantum Dye or its conjugates. Under these conditions, in the presence of the gadolinium-thenoyltrifluoroacetonate complex as the energy transfer species, the luminescence of the europium Quantum Dye increased approximately 6-fold upon drying. However, the presence of a nonemitting lanthanide such as gadolinium is not always required for this effect. In studies employing the 2,6-pyridinedicarboxylate ion as the energy transfer species, where both the terbium and the europium Quantum Dyes could be simultaneously excited at $280 \mathrm{~nm}$, the presence of gadolinium actually decreased the luminescence compared to that obtained with the 2,6-pyridinedicarboxylate alone. The simplest explanation for the FRETEL effect is that fluorescence resonance energy transfer occurs between the photo-trapping energy transfer species, either unbound or complexed with the nonluminescent gadolinium ion. The energy being finally transferred to the luminescent lanthanide ion complexes with consequent increase in emission intensity. This new method for the enhancement of luminescence intensity in the solid state has the significant advantage of eliminating the need for the previously required aqueous emulsion, which was difficult to make and transport.
\end{abstract}

\section{INTRODUCTION}

For use as labels, the luminescent complexes of trivalent lanthanide ions offer many advantages compared to quantum dots and conventional fluorophores. These advantages include narrower emission bands, the capacity for inexpensive time-gating, small size compared to nanoparticles, and the potential for a high degree of multiple labeling because of the small size and absence of concentration quenching. Furthermore, the Quantum Dyes are ideal for ratiometric measurements because complexes of different luminescent lanthanide ions with the same organic ligand(s) have virtually identical chemical properties and solubility patterns, but their emissions do not overlap. Thus, non-interfering simultaneous detection of different targets is possible.

Lanthanide ions and their complexes, however, have one major deficiency--their molar absorptivities are low (less than $\left.10 \mathrm{M}^{-1} \mathrm{~cm}^{-1}\right)^{1}$ compared to those of highly absorbing organic fluorophores, such as cyanine dyes, or of quantum dots. This very low absorptivity is mitigated by the use of organic ligands that absorb light and then transfer energy directly to the emitting lanthanide ion ${ }^{2}$, and by the use of energy acceptor-donor (columinescence) complexes that ultimately transfer energy to the emitting lanthanide ion ${ }^{3}$. In the following, the term energy transfer species will be used to describe both these organic ligands and the accepter-donor complexes. The absorptivity of these energy transfer species is sufficient to permit the successful use of lanthanide complexes for luminescent assays. For example, the absorptivities (molar extinction coefficients) of the sodium salt and $\mathrm{Gd}^{3+}$ complex of the anion (TTFA) of 4,4,4-trifluoro-1(2-thienyl)-1,3-butanedione in ethanol solution are 15,280 and 47,040, respectively, when measured at the absorption maximum (340 nm), and 7,120 and 25,960, respectively, when measured at the wavelength, $365 \mathrm{~nm}$, presently used for their excitation. By comparison, the molar extinction coefficient of $\mathrm{Cy} 3$ has been reported ${ }^{4}$ to be 150,000 at $550 \mathrm{~nm}$.

$\mathrm{Xu}$ and $\mathrm{Hemmila}^{3}$ circumvented the problem of ligands with low extinction coefficients by taking advantage of energy transfer in a micellar solution from complexes of a non-emitting $\mathrm{Ln}^{3+}$ to complexes of an emitting $\mathrm{Ln}^{3+}$. Since the concentration of the non-emitting $\mathrm{Ln}^{3+}$ was greater than that of the emitting $\mathrm{Ln}^{3+}$, any of several non-emitting $\mathrm{Ln}^{3+}$ complexes could ultimately transmit energy to an emitting $\mathrm{Ln}^{3+}$ complex. Under these conditions, the effective absorptivity *rleif@rleif.com; phone 1619 582-0437; www.newportinstruments.com

Ultrasensitive and Single-Molecule Detection Technologies, edited by Jörg Enderlein, Zygmunt K. Gryczynski, Proc. of SPIE Vol. 6092, 609206, (2006) - 1605-7422/06/\$15 - doi: 10.1117/12.645069 
would be proportional to the sum of the absorptivities of all the non-emitting $\mathrm{Ln}^{3+}$ complexes in the vicinity of an emitting $\mathrm{Ln}^{3+}$ complex. This columinescence process was extended by Leif and Vallarino to macromolecules labeled with the europium Quantum Dye ${ }^{5,6}$. The importance of micellar organization for the enhanced luminescence composition was demonstrated by the observation ${ }^{7}$ that a water-miscible polar solvent such as ethanol, when added to the characteristically cloudy and luminous micellar composition, completely eliminated the luminescence enhancement while simultaneously turning the cloudy micellar liquid to a clear solution. A simple explanation for this effect, suggested by Tong et al. ${ }^{8}$, is that only in the micelles the concentration of $\operatorname{Gd}(\mathrm{TTFA})_{3}$ complexes present within the Förster distance of the europium macrocycle units was sufficiently high to permit extensive energy transfer.

Further studies established that columinescence could occur in the solid state, including fixed, stained, and mounted microscope slide preparations ${ }^{9}$. In studies involving the use of streptavidin conjugates of the europium Quantum Dye (EuMac), it was determined ${ }^{9}$ that the luminescence enhancement obtained by the addition of Gd(TTFA) 3 was 4.6 times greater than that obtained by the addition of a threefold molar amount of HTTFA (4,4,4-trifluoro-1(2-thienyl)-1,3-butanedione, otherwise known as thenoyltrifluoroacetone), which provided the same quantity of HTTFA molecules as the TTFA ligands of the gadolinium complex. However, it was also observed ${ }^{10}$ that under certain conditions the presence of the gadolinium ion actually decreased the luminescence relative to that obtained in the presence of only the sodium salt, NaTTFA. Since it appears that fluorescence resonance energy transfer (FRET) is involved in this luminescence enhancement process, the generic term fluorescence resonance energy transfer enhanced luminescence (FRETEL) is suggested. A possible mechanism for the energy transfer from excess ligands or fluorophores to complexed lanthanide ions emitters in the solid-state will be suggested.

\subsection{STARTING MATERIALS}

The unfunctionalized europium Quantum Dye (EuMac-un), unfunctionalized terbium Quantum Dye (TbMac-un), europium Quantum Dye conjugate of streptavidin (EuMac-Strept), Gd(TTFA) ${ }_{3}$, NaTTFA, and $\mathrm{Na}_{3} \mathrm{Gd}(\mathrm{PDCA})_{3}$ were produced by the Newport Instruments-Virginia Commonwealth University collaborative effort. Sodium hydroxide, ACS Grade, was purchased from EM Science, Affiliate of Merck KGaA, Darmstad, Germany, (Catalog No. SX 0590-1). 2,6pyridinedicarboxylic acid, $\mathrm{H}_{2}$ PDCA, was purchased from Aldrich (Catalog No. P.6, 380-8). Parafilm 4 in. x $125 \mathrm{ft}$. roll (Laboratory Film) was purchased from Pechiney Plastic Packaging, Menasha, WI, (Catalog No. PM-996). Reacti-Bind Biotin Coated Microwell Strip Plates were purchased from Pierce Biotechnology, Inc., Rockford, IL, (Catalog No. 15151). White, "U" bottomed, 96 well, microtiter plates were purchased from Thermo Electron Corp. (Franklin, MA, part no. 7105). Binder-free Glass Microfibre Filter sheet $460 \times 570 \mathrm{~mm}$. $(1.2 \mu \mathrm{m}$ porosity, GF/C grade) was purchased from Whatman, Cat. No. 1822-914 (www.whatman.com).

\subsection{SOLUTIONS}

TBS-Azide concentrate is an aqueous solution that contains in 1 liter: $12.12 \mathrm{~g}$ of TRIS, $87.7 \mathrm{~g}$ of NaCl, and $5.0 \mathrm{~g}$ of $\mathrm{NaN}_{3}$. TBS-Azide $\mathrm{pH} 7.0$ and TBS-Azide $\mathrm{pH} 7.4$ are tenfold dilutions of this concentrate, with the $\mathrm{pH}$ lowered by the addition of $1 \mathrm{M} \mathrm{HCl}$. Similarly, TBS-Azide $\mathrm{pH} 8.5$ is a tenfold dilution of the concentrate, with the $\mathrm{pH}$ raised by the addition of $1 \mathrm{M}$ $\mathrm{NaOH}$. A stock $(1 \mathrm{mg} / \mathrm{mL})$ solution of the EuMac-Strept in TBS Azide $\mathrm{pH} 7.4$ was diluted with TBS-Azide to $0.1 \mathrm{mg} / \mathrm{mL}$. BSA pH 7.0 is a solution that contains $0.5 \% \mathrm{w} / \mathrm{v}$ BSA in TBS-Azide $\mathrm{pH}$ 7.0. Similarly, BSA pH 8.5 is a solution that contains $0.5 \% \mathrm{w} / \mathrm{v}$ BSA in TBS-Azide $\mathrm{pH}$ 8.5. A $10,000 \mu \mathrm{M}(10 \mathrm{mM})$ stock solution of $\mathrm{Gd}(\mathrm{TTFA})_{3}$ in ethanol was prepared by dissolving $8.2 \mathrm{mg}$ of $\mathrm{Gd}(\mathrm{TTFA})_{3}$ in $1.0 \mathrm{~mL}$ of ethanol. This solution was serially diluted tenfold with ethanol to produce $1000 \mu \mathrm{M}, 100 \mu \mathrm{M}$, and $10.0 \mu \mathrm{M} \mathrm{Gd(TTFA})_{3}$ ethanolic solutions. A $30 \mathrm{mM}$ stock ethanolic solution of NaTTFA was prepared by dissolving $8.6 \mathrm{mg}$ of NaTTFA in $1.174 \mathrm{~mL}$ of ethanol. This $30 \mathrm{mM}$ NaTTFA solution was serially diluted tenfold with ethanol to produce $3000 \mu \mathrm{M}, 300 \mu \mathrm{M}$, and $30.0 \mu \mathrm{M}$ NaTTFA ethanolic solutions. Each NaTTFA ethanolic solution had the same TTFA anion concentration as one of the $\operatorname{Gd}(T T F A)_{3}$ ethanolic solutions. Equal volumes of the Gd(TTFA $)_{3}$ and NaTTFA ethanolic solutions, and each of their dilutions having the same TTFA anion concentrations, were mixed together to produce corresponding Gd+NaTTFA ethanolic solutions with the same series of concentrations.

A $30 \mathrm{mM}$ stock solution of HTTFA was prepared by dissolving $9.2 \mathrm{mg}$ of HTTFA in $1.38 \mathrm{~mL}$ of ethanol. The $30 \mathrm{mM}$

Proc. of SPIE Vol. 6092 609206-2 
stock solution of HTTFA was serially diluted tenfold with ethanol to produce $3000 \mu \mathrm{M}, 300 \mu \mathrm{M}$, and $30.0 \mu \mathrm{M}$ HTTFA ethanolic solutions. Each HTTFA ethanolic solution had the same concentration of energy transfer species as its corresponding Gd(TTFA) ${ }_{3}$ ethanolic solution, except for the energy transfer species being in the molecular HTTFA form instead of the mono-negative anionic TTFA form. The Gd+HTTFA solutions were prepared by mixing equal volumes of the $\operatorname{Gd}(\text { TTFA })_{3}$ and HTTFA ethanolic solutions. The Na+HTTFA solutions were prepared by mixing equal volumes of the NaTTFA and HTTFA ethanolic solutions.

A $5.0 \times 10^{-3} \mathrm{M}$ solution of $\mathrm{Na}_{2} \mathrm{PDCA}$ in methanol was prepared as follows: $\mathrm{H}_{2}$ PDCA (167.7 mg, $\left.1.00 \mathrm{mmol}\right)$ was dissolved in $25.0 \mathrm{~mL}$ of methanol to give a $4.03 \times 10^{-2} \mathrm{M}$ solution. $\mathrm{NaOH}(167.9 \mathrm{mg}, 4.20 \mathrm{mmol})$ was dissolved in $100.0 \mathrm{~mL}$ of methanol to give a $4.20 \times 10^{-2} \mathrm{M}$ solution. Then $1.25 \mathrm{~mL}$ of the $\mathrm{H}_{2} \mathrm{PDCA}$ solution and $2.50 \mathrm{~mL}$ of the $\mathrm{NaOH}$ solution were mixed and the volume was brought up to $10.0 \mathrm{~mL}$ with methanol. A $5.0 \times 10^{-4} \mathrm{M}$ methanolic solution of $\mathrm{Na}_{2} \mathrm{PDCA}$ was prepared by diluting tenfold with methanol the $5.0 \times 10^{-3} \mathrm{M} \mathrm{Na}_{2}$ PDCA methanolic solution. A $10.5 \mathrm{mM}$ stock solution of EuMac-un in methanol was prepared by adding $18.8 \mathrm{mg}$ of EuMac-un to $2.5 \mathrm{~mL}$ of methanol. This solution was serially diluted with methanol to produce a $10.5 \mu \mathrm{M}$ solution of EuMac-un. A $10.8 \mathrm{mM}$ stock solution of the TbMac-un was prepared by adding $18.9 \mathrm{mg}$ of TbMac-un to $2.5 \mathrm{~mL}$ of methanol. This solution was serially diluted with methanol to produce a $10.8 \mu \mathrm{M}$ solution of TbMac-un. A $3.48 \times 10^{-3} \mathrm{M}$ solution of $\mathrm{Na}_{3} \mathrm{Gd}(\mathrm{PDCA})_{3}$, equivalent to a $1.04 \times 10^{-2} \mathrm{Eqv} / \mathrm{L}$ solution of PDCA anions, was prepared by dissolving $62.8 \mathrm{mg}$ of $\mathrm{Na}_{3} \mathrm{Gd}(\mathrm{PDCA})_{3}$ in $25 \mathrm{~mL}$ of methanol. This solution was diluted tenfold with methanol to produce a $3.48 \times 10^{-4} \mathrm{M}$ solution of $\mathrm{Na}_{3} \mathrm{Gd}(\mathrm{PDCA})_{3}$, equivalent to a $1.04 \times 10^{-3}$ Eqv/L solution of PDCA anions.

\subsection{PREPARATION OF MICROWELL STRIP PLATES}

EuMac labeled and control microwell strip plates were prepared as follows. One hundred $\mu \mathrm{L}$ of BSA pH 8.5 was added, as a rinse, to each of the wells of Reacti-Bind Biotin Coated Microwell Strip Plates at room temperature $\left(26{ }^{\circ} \mathrm{C}\right)$ and the supernatant was removed by aspiration. A second $100 \mu \mathrm{L}$ of BSA pH 8.5 was added to each well. The wells were incubated for $15 \mathrm{~min}$. at room temperature and the supernatant was removed by aspiration. Just prior to use, the $0.05 \mathrm{mg} / \mathrm{mL}$ EuMac-Streptavidin solution was diluted with the BSA pH 7.0 solution to a final concentration of $0.5 \mu \mathrm{g} / \mathrm{mL}$, and $50 \mu \mathrm{L}$ of the resulting solution was pipetted into each of eight biotinylated wells. An equal number of control wells did not receive the EuMac-Strept solution. Then, the microtiter strip was covered with Parafilm to prevent evaporation and incubated at room temperature and in the dark for approximately $30 \mathrm{~min}$. The supernatants were removed and the EuMacStrept and control wells were washed 3 times with $100 \mu \mathrm{L}$ of BSA pH 7.0, which was removed by aspiration. The wells were allowed to dry at room temperature.

\subsection{OUTLINE OF THE EXPERIMENTS}

Two sets of studies were performed to investigate the effect of the concentration of the energy transfer donor. The first study involved the treatment of the EuMac-Streptavidin-coated wells with ethanolic solutions containing TTFA and HTTFA in various forms; the second involved the addition to microtiter plates of methanolic solutions of $\mathrm{Na}_{2} \mathrm{PDCA}$ or $\mathrm{Na}_{3} \mathrm{Gd}(\mathrm{PDCA})_{3}$, together with either the unfunctionalized EuMac (EuMac-un) or the unfunctionalized TbMac (TbMacun).

In the first set of experiments, $30 \mu \mathrm{L}$ of the serially diluted solutions of the energy transfer species were added in a sequential manner to pairs of control and EuMac-Streptavidin-coated wells. The microtiter strips were allowed to air dry overnight in the dark and the luminescence was measured as follows. The microtiter strips were placed into a UVP Epi Chem II Darkroom and illuminated with the long UV (ca. $365 \mathrm{~nm}$ ) bulb; the resulting emission was passed through a 619 $\mathrm{nm}$ filter. Digital images of the strips were acquired with a cooled Quantitative Imaging Co. Retiga EX camera (www.qimaging.com). The images were binned to $340 \times 259$ pixels. The image of the center $81 \%$ of the well was analyzed. The average emission intensity was calculated with Fovea PhotoShop plug-in (http://www.reindeergraphics.com) under Filter/IP*Features/Regions.

The second set of studies involved both the Eu/Mac-un and the TbMac-un. One set of EuMac-un methanolic solutions and one set of TbMac-un methanolic solutions were each mixed with the various concentrations of $\mathrm{Na}_{2} \mathrm{PDCA}$; another set 
of EuMac-un and TbMac-un methanolic solutions were each mixed with the various concentrations of $\mathrm{Na}_{3} \mathrm{Gd}(\mathrm{PDCA})_{3}$. The resulting mixtures were added to microtiter plates. In the mixtures, the concentrations of the EuMac-un and TbMacun were respectively 5.05 and $5.15 \mu \mathrm{M}$. The concentrations of $\mathrm{Na}_{2}$ PDCA ranged from 0.00 to $752 \mu \mathrm{M}$, and those of $\mathrm{Na}_{3} \mathrm{Gd}(\mathrm{PDCA})_{3}$ ranged from 0.00 to $2,080 \mu \mathrm{Eqv} / \mathrm{L}$ of PDCA anion $(693 \mu \mathrm{M})$. For the $\mathrm{Na}_{3} \mathrm{Gd}(\mathrm{PDCA})_{3}$ solutions, the concentration expressed as Eqv/L of PDCA anion is equal to three times the concentration expressed as molarity of $\mathrm{Na}_{3} \mathrm{Gd}(\mathrm{PDCA})_{3}$ complex. Equal volumes $(250 \mu \mathrm{L})$ of each series of solutions were added to individual wells of the $\mathrm{U}$ bottom plates. The solutions were allowed to dry by storing the plates at room temperature, in the dark, for $24 \mathrm{hr}$. The dry plates were mounted on the Cary Eclipse microplate reader and scanned. The emission spectra of the solid residues were obtained with a Varian Cary fluorometer operated in time-gated luminescence mode with a delay of $100 \mu \mathrm{sec}$. Fifty emission spectra were summed to produce the final spectrum. The excitation and emission slits were respectively 10 and 2.5 nm.

A third series of experiments was performed to investigate the increase in luminescence during drying. Fiberglass disks 6 $\mathrm{mm}$ in diameter were fabricated from a Glass Microfibre Filter sheet using a single hole paper punch. Chamber slides were made by placing a $6 \mathrm{~mm}$ fiberglass disc on a microscope slide and straddling it on both sides in the long direction by $3 \mathrm{M}$ double stick tape. After a sample was added to the disk, the chamber was closed by a coverglass. Subsequenly, $8 \mu \mathrm{L}$ of an ethanolic solution of $1 \mathrm{mM} \mathrm{Gd(TTFA})_{3}$ was applied to the exposed edge of the disk; and the flooded disk was allowed to dry slowly.

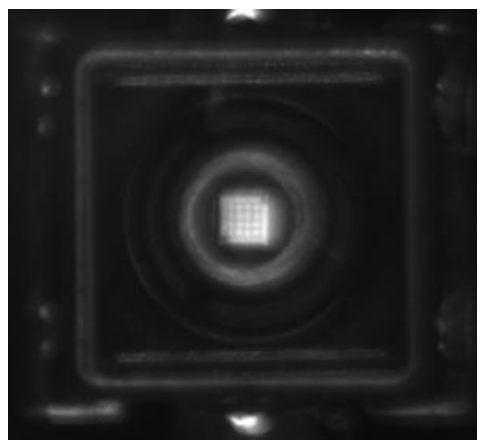

Figure 1. Image of a Nichia UV LED, MODEL NCCU033

(T).The square chip is approximately $6.3 \mathrm{~mm}$ on a side. The small (1 $\mathrm{mm}$ on a side) white checker-board-appearing area in the center shows the light emitted by the chip.
The luminescence was measured with a modified epi-illuminated microscope, equipped with a $20 \mathrm{x}$ objective with a N.A. of 0.50 . The image of the same central portion of the luminescent spot was analyzed for all measurements. The light source was a Nichia UV LED, Model NCCU033. According to the manufacturer's specifications, the peak wavelength, optical power output, and spectrum half width were respectively $365 \mathrm{~nm}, 100 \mathrm{~mW}$, and 10 $\mathrm{nm}$. The emission pattern of the UV LED (Figure 1), unlike that of an arc or a flashlamp, is not a point source; it is an array. A Linos condenser combina-

tion (16/21.4 mm) (part num. 06 3010, http://www.linos-photonics.com) was attached to the back of the epi-illuminator, as shown in Figure 2A. The heat sink at the rear of the UV LED was attached to a threaded brass pipe (Figure 2B), positioned by a previously described flashlamp assembly ${ }^{11}$ that replaced the light-source assembly of the microscope. A Laserlab supply (http://www.laserlab.com/) was used to drive the LED in pulsed mode. One ms wide pulses were delivered at $10 \mathrm{~Hz}$. The images were triggered by the trailing edge of the pulses and had an exposure time of $2 \mathrm{~ms}$. The approximate $9 \mu$ s turn-on time of the camera ensured that the background fluorescence of the sample had totally decayed before the emission was measured. Three hundred frames were summed by the Retiga EX camera and saved as raw 32 bit images with the QImaging QCapture Suite. The use of 32 bit images eliminated the possibility of the summation resulting in a numerical overflow. An image of the background with the light path to the camera blocked was subtracted from the summed image. Since some of the pixels in these summed images (particularly those with weak emissions) had lower values than the corresponding pixels of the background images, a summation calculation was included in the subtraction operation. Negative values produced by the subtraction were included in the sum but changed to zero for the resultant image, which was then rescaled for viewing to 8 bits. Custom software for these operations was written in the Ada programming language. The luminous flux density on a surface produced by the UV LED was, conservatively, 85 times greater than that produced by the fluorescent lamp employed for the FRETEL effect studies with the UV light box described above. 

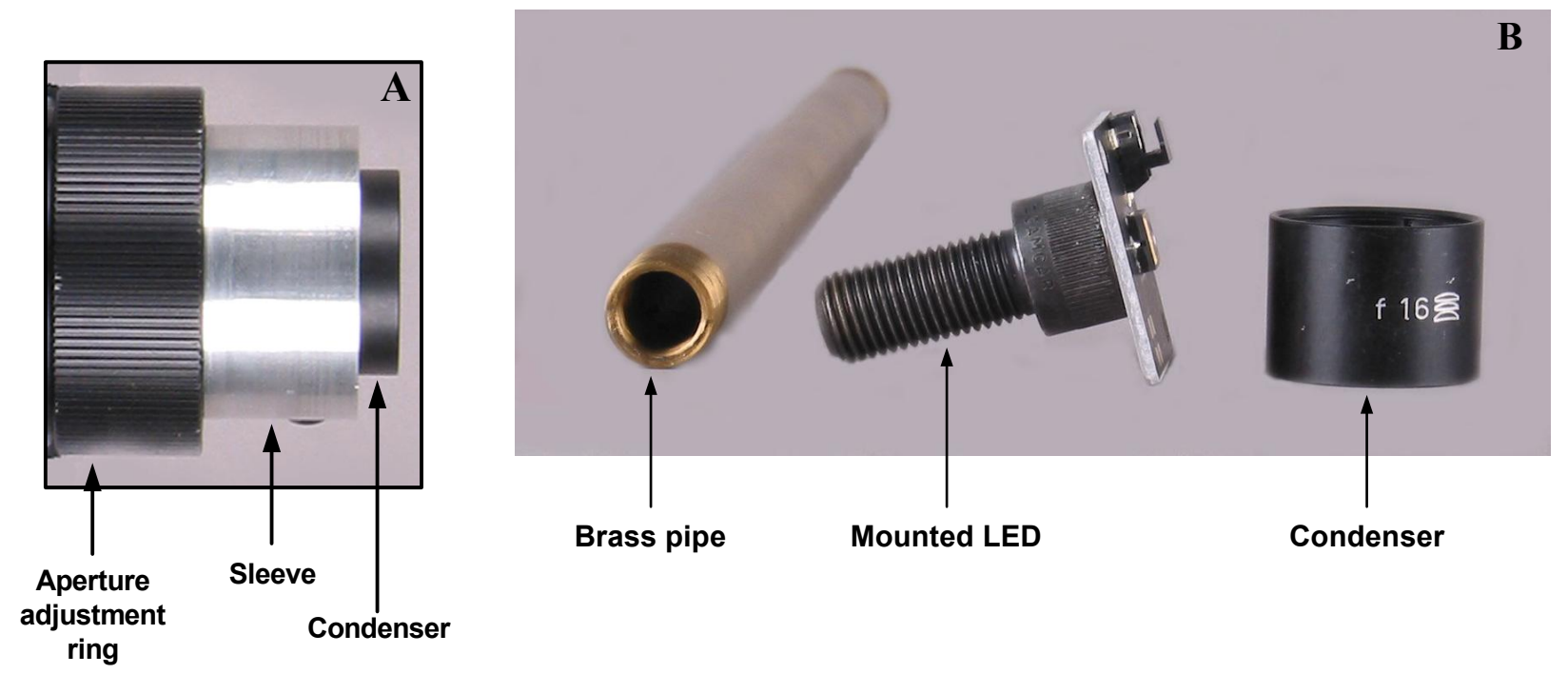

Figure 2.A, The condenser is mounted in an aluminum sleeve, which is secured to the aperture adjustment assembly of the epiilluminator. The excitation light enters from the right. B, The LED is mounted on a screw that is screwed into the front end of the brass pipe. A side view of the condenser is shown on the right.

\subsection{EuMac-Streptavidin Studies}

The effects of Gd(TTFA) ${ }_{3}$ and NaTTFA on the luminescence of the EuMac-Strept and control preparations were determined as described for the first series of studies outlined in Section 2.4. The luminescence from 3 sets of serial dilutions were measured. The first set consisted of $1,000 \mu \mathrm{M}, 100 \mu \mathrm{M}$, and $10.0 \mu \mathrm{M} \mathrm{Gd}(\mathrm{TTFA})_{3}$ ethanolic solutions, the second of $3,000 \mu \mathrm{M}, 300 \mu \mathrm{M}$, and $30.0 \mu \mathrm{M}$ NaTTFA ethanolic solutions, and the third of 3,000 $\mu$ Eqv/L, $300 \mu \mathrm{Eqv} / \mathrm{L}$, and 30.0 $\mu$ Eqv/L Gd+NaTTFA ethanolic solutions. Thus, 6 sets of data were measured: Gd(TTFA) $)_{3}$ added to EuMac-Strept coated wells (EuMac-Strept+Gd(TTFA) ${ }_{3}$ ), Gd(TTFA) ${ }_{3}$ added to control wells (Ctrl. Gd(TTFA) $)_{3}$, NaTTFA added to EuMacStrept coated wells (EuMac-Strept+NaTTFA), NaTTFA added to control wells (Ctrl. NaTTFA), Gd+NaTTFA added to EuMac-Strept coated wells (EuMac-Strept+ Gd+NaTTFA), and Gd+NaTTFA solutions added to control wells (Ctrl. Gd+NaTTFA).

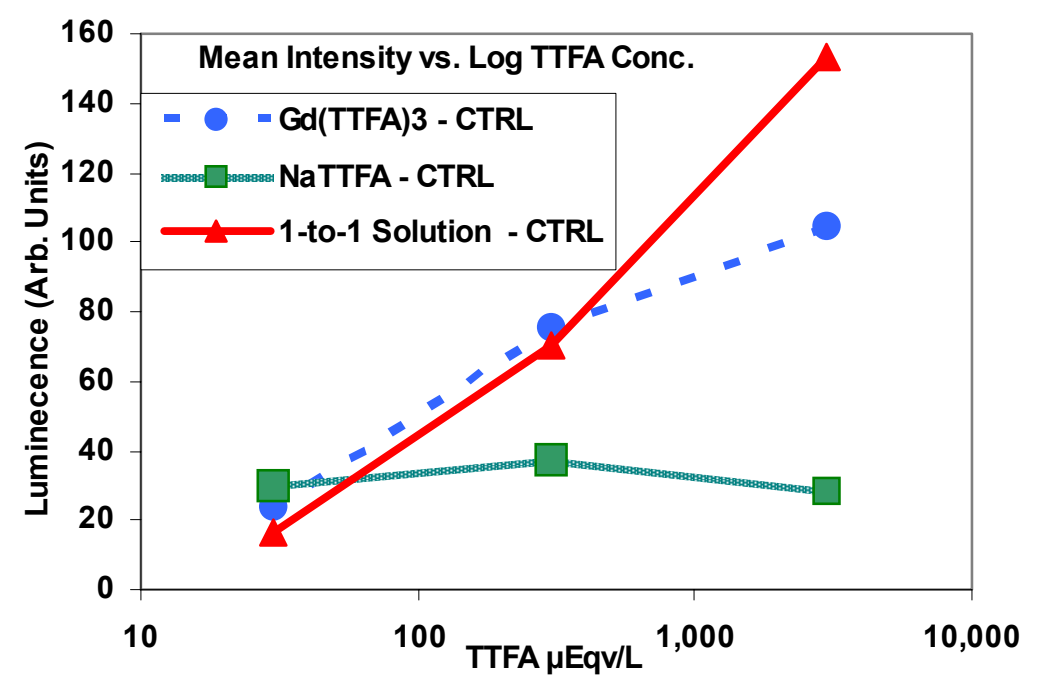

Figure 3 is a plot of the corrected luminescence of EuMac-Strept. vs. the concentrations, on a logarithmic scale, of the energy transfer species Gd(TTFA) $)_{3}$, NaTTFA, and their one-to-one mixtures. At the highest concentration of added energy transfer species, the EuMac-Strept. with the one-to-one mixture is brighter than that with the Gd(TTFA) ${ }_{3}$. 
The luminescence data are shown in Figure 3 and in Tables 1a and 1b. In Table 1a, the results for the wells treated with the Gd(TTFA) $)_{3}$ solutions are shown on the left and those for the wells treated with the NaTTFA solutions on the right. The results for the wells treated with the Gd+NaTTFA solutions are shown in Table 1b. After the luminescence from the control was subtracted (-Ctrl.) from that of the sample, the maximum net luminescence of the EuMac-Strept coated wells occurred at the highest concentration, $1,000 \mu \mathrm{M}(3,000 \mu \mathrm{Eqv} / \mathrm{L})$, for the $\mathrm{Gd}(\mathrm{TTFA})_{3}$ and $\mathrm{Gd}+\mathrm{NaTTFA}$ solutions; whereas, it occurred at the second highest concentration, $300 \mu \mathrm{Eqv} / \mathrm{L}$, for the NaTTFA solution. For each data point, the concentration of the TTFA anions shown on the abscissa of Figure 3 was threefold the concentration of the Gd(TTFA) 3 . An inner filter effect is a possible explanation for the quenching of the luminescence at the highest concentration of NaTTFA.

Table 1a. Luminescence (Arb. units)

\begin{tabular}{|r|r|r|r||r|r|r|r|}
\hline $\begin{array}{c}\text { Gd } \\
(\mathrm{TTFA})_{3} \\
(\mu \mathrm{M})\end{array}$ & $\begin{array}{c}\text { EuMac- } \\
\text { Strept }+ \\
\text { Gd } \\
(\text { TTFA })_{3}\end{array}$ & $\begin{array}{c}\text { Ctrl. Gd } \\
(\text { TTFA })_{3}\end{array}$ & $\begin{array}{c}\text { EuMac- } \\
\text { Strept }+ \\
\text { Gd } \\
(\text { TTFA })_{3} \\
- \text { Ctrl. }\end{array}$ & $\begin{array}{c}\text { Na } \\
\text { TTFA } \\
(\mu \mathrm{M})\end{array}$ & $\begin{array}{c}\text { EuMac- } \\
\text { Strept + } \\
\mathrm{Na} \\
\text { TTFA }\end{array}$ & $\begin{array}{c}\text { Ctrl. } \\
\mathrm{Na} \\
\text { TTFA }\end{array}$ & $\begin{array}{c}\text { EuMac- } \\
\text { Strept + } \\
\mathrm{Na} \\
\text { TTFA } \\
- \text { Ctrl. }\end{array}$ \\
\hline 1,000 & 136.9 & 32.2 & 104.7 & 3,000 & 65.9 & 37.7 & 28.3 \\
\hline 100 & 100.5 & 25.3 & 75.5 & 300 & 61.8 & 24.8 & 37.1 \\
\hline 10 & 45.6 & 21.7 & 24.0 & 30 & 52.6 & 23.0 & 29.6 \\
\hline
\end{tabular}

Table 1b. Luminescence (Arb. units)

\begin{tabular}{|r|r|r|r|r|}
\hline $\begin{array}{c}\text { Gd } \\
(\mathrm{TTFA})_{3} \\
(\mu \mathrm{M})\end{array}$ & $\begin{array}{c}\text { Na } \\
\text { TTFA } \\
(\mu \mathrm{M})\end{array}$ & $\begin{array}{c}\text { EuMac- } \\
\text { Strept } \\
\text { Gd+NaTTFA } \\
\text { Solution }\end{array}$ & $\begin{array}{c}\text { Ctrl. } \\
\text { Gd+NaTTFA } \\
\text { Solution }\end{array}$ & $\begin{array}{c}\text { EuMac- } \\
\text { Strept + } \\
\text { Gd+NaTTFA } \\
\text { Solution } \\
- \text { Ctrl. }\end{array}$ \\
\hline 500 & 1,500 & 188.0 & 34.9 & 153.1 \\
\hline 50 & 150 & 100.6 & 30.7 & 69.9 \\
\hline 5 & 15 & 43.7 & 27.8 & 16.1 \\
\hline
\end{tabular}

In the solid phase, the ratio (3.7) between the luminescence intensity of the EuMac-Strept samples with Gd(TTFA) ${ }_{3}$ and the EuMac-Strept samples with NaTTFA, both at the same total concentration of TTFA anions $(3,000 \mu \mathrm{Eqv} / \mathrm{L})$, shows (Table 1a) the enhancement caused by gadolinium to be lower than previously observed ${ }^{9}$. The ratio was even lower (2.8) when measured at the highest net luminescence value of the EuMac-Strept samples with NaTTFA, corresponding to a TTFA concentration of $300 \mu \mathrm{Eqv} / \mathrm{L}$. And, at the highest concentrations of the $\mathrm{Gd}(\mathrm{TTFA})_{3}$ solutions (Table 1a) and Gd+NaTTFA solutions (Table 1b), the ratio of the luminescence intensities (104.7/153.1) was 0.7. Thus, under some conditions, lowering the concentration of the Gd(III) ion can increase the luminescence intensity. Since NaTTFA has been shown to be an effective energy transfer species of luminescence, the ratio between the increase in luminescence at 3,000 $\mu \mathrm{Eqv} / \mathrm{L}$ and at $30 \mu \mathrm{Eqv} / \mathrm{L}$ may provide a true measure of the FRETEL effect. For Gd(TTFA) ${ }_{3}$, NaTTFA, and the 1-to-1 solutions, these ratios were 4.4, 1.0, and 9.5, respectively. 
Bromm et al. ${ }^{12}$ demonstrated that the presence of Gd(III) with a EuMac-avidin conjugate in a micellar solution resulted in a luminescence "more than ten times higher than that of the other solutions". The simplest explanation for the higher luminescence intensity ratio (10 vs. 3.7) of micellar solutions compared to the system considered above (Table 1a) is that the solid phase environment allows a much more effective resonance energy $\operatorname{transfer}^{13}$ by the excess TTFA anions, which are neither complexed with the gadolinium ion nor bound to Eu macrocycles. Therefore, for the data of Table 1a, the ratio between the luminescence with Gd(TTFA) $)_{3}$ and the luminescence with NaTTFA alone is artificially decreased because of the increased luminescence enhancement caused by NaTTFA in the solid phase.

The effect of ethanolic solutions of Gd(TTFA) ${ }_{3}$, NaTTFA, HTTFA, and mixtures thereof on the luminescence of the EuMac-Strept and control preparations were determined as described for the first series of studies outlined in Section 2.4. The luminescence from 6 sets of serial dilutions were measured. The first set consisted of 10,000 $\mu \mathrm{M}, 1,000 \mu \mathrm{M}, 100 \mu \mathrm{M}$, and $10.0 \mu \mathrm{M} \mathrm{Gd}(\mathrm{TTFA})_{3}$ one-component ethanolic solutions. The second and third sets consisted of NaTTFA and HTTFA one-component ethanolic solutions, with concentrations decreasing in the order $30,000 \mu \mathrm{M}, 3,000 \mu \mathrm{M}, 300 \mu \mathrm{M}$, and $30 \mu \mathrm{M}$. (Each of these solutions contained the same concentration of TTFA units as the corresponding Gd(TTFA) $)_{3}$ solution of the first set, because there are 3 TTFA anions in each Gd(III) complex.) The Na+HTTFA solutions (4th set) consisted of equal volumes of the NaTTFA and the HTTFA ethanolic solutions. The Gd+HTTFA solutions (5th set) consisted of equal volumes of the Gd(TTFA) $)_{3}$ and HTTFA ethanolic solutions. And the Gd+NaTTFA solutions (6th set) consisted of equal volumes of the Gd(TTFA $)_{3}$ and NaTTFA ethanolic solutions. For each of these one-to-one solutions, the concentrations of the TTFA anion, of the HTTFA molecule, or of their sum were $30,000 \mu \mathrm{Eqv} / \mathrm{L}, 3,000 \mu \mathrm{Eqv} / \mathrm{L}, 300$ $\mu \mathrm{Eqv} / \mathrm{L}$, and $30 \mu \mathrm{Eqv} / \mathrm{L}$, respectively. For these studies, $30 \mu \mathrm{L}$ aliquots of each solution were added to two EuMacStreptavidin-coated wells, and a third $30 \mu \mathrm{L}$ aliquot was added to a control well.

The data points from the two EuMac-Streptavidin-coated wells of each set were averaged and the luminescence from the control well was subtracted. These averaged net results are shown in Table 2 and Figure 4. In Table 2, the results for the wells treated with the one-component solutions are shown on the left, and those for the wells treated with the one-to-one solutions are shown on the right.

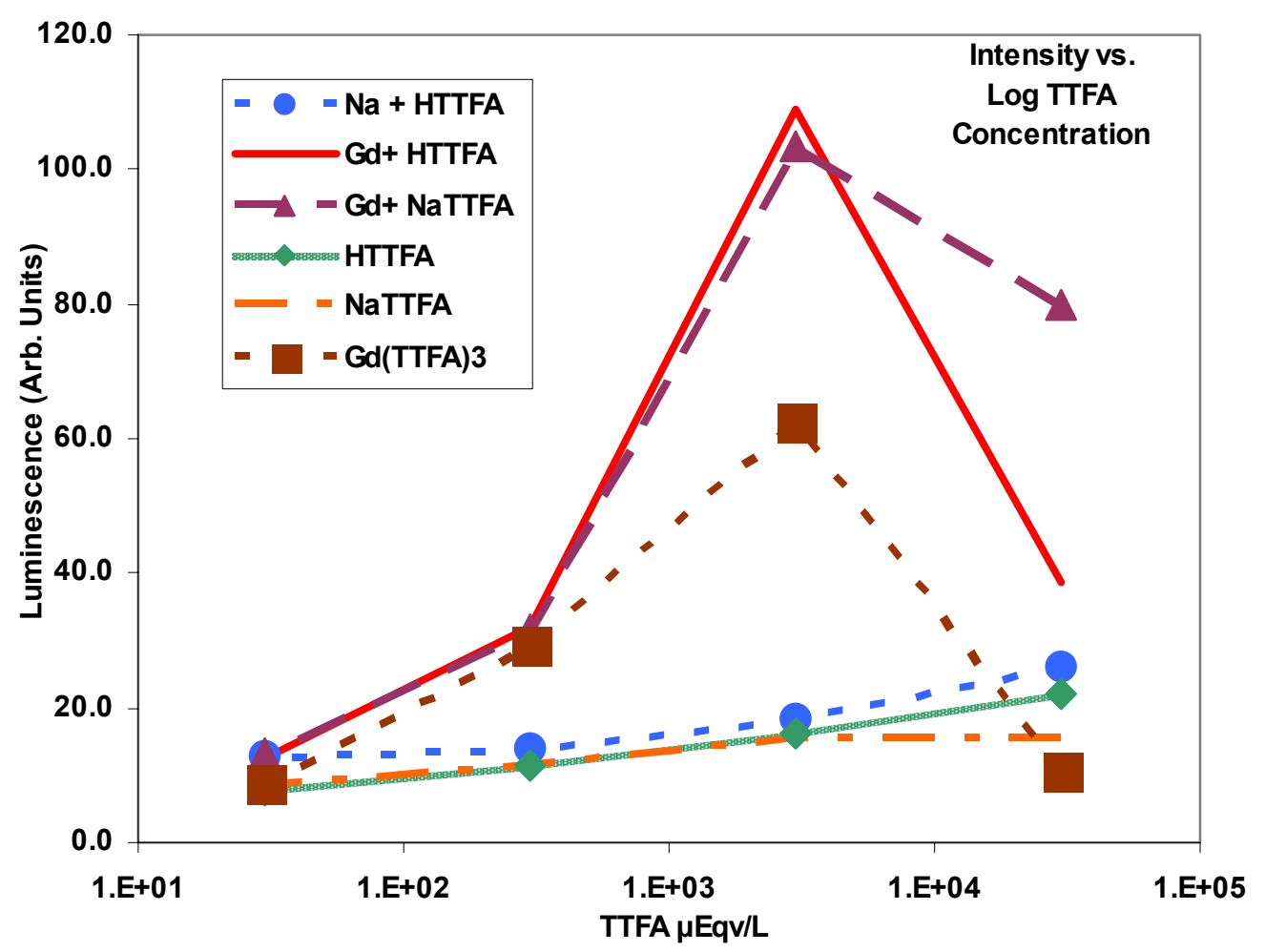

Figure 4 is a plot of the concentrations, on a logarithmic scale, of the energy transfer species Gd(TTFA) ${ }_{3}$, NaTTFA, HTTFA, and their mixtures vs. the luminescence of the EuMac-Streptavidin. 
Table 2. Luminescence (Arb. units)

\begin{tabular}{|r|r|r|r|r|r|r|r|}
\hline & \multicolumn{3}{|c|}{ One-Component Solutions } & \multicolumn{2}{c|}{ One-to-One Solutions } & \\
\hline $\begin{array}{r}\text { TTFA } \\
\mu \mathrm{EFv} / \mathrm{L}\end{array}$ & HTTFA & $\begin{array}{c}\mathrm{Na} \\
\text { TTFA }\end{array}$ & $\begin{array}{c}\text { Gd } \\
\text { (TTFA })_{3}\end{array}$ & $\begin{array}{c}\mathrm{Na} \\
+ \\
\text { HTTFA }\end{array}$ & $\begin{array}{c}\text { Gd } \\
+ \\
\text { HTTFA }\end{array}$ & $\begin{array}{c}\text { Gd } \\
+ \\
\text { NaTTFA }\end{array}$ & $\begin{array}{c}\text { TTFA } \\
\text { nEqv } \\
\text { per well }\end{array}$ \\
\hline 30,000 & 22.0 & 16.0 & 10.6 & 26.0 & 38.7 & 80.0 & 900 \\
\hline 3,000 & 16.0 & 15.8 & 62.5 & 18.3 & 109.0 & 103.3 & 90 \\
\hline 300 & 11.3 & 11.7 & 29.6 & 14.2 & 32.1 & 31.8 & 9.0 \\
\hline 30 & 7.9 & 8.7 & 8.8 & 12.7 & 12.7 & 13.3 & 0.90 \\
\hline
\end{tabular}

The maximum net luminescence occurred at the second highest concentration, 3,000 $\mu \mathrm{Eqv} / \mathrm{L}$, for both one-to-one solutions containing $\mathrm{Gd}(\mathrm{III})$, namely the $\mathrm{Gd}+\mathrm{HTTFA}$ and the $\mathrm{Gd}+\mathrm{NaTTFA}$ solutions. The difference between the two values (109.0 and 103.3) is within the error of the experiment. Both of these values are greater than the maximum value (62.5) for the one-component $\operatorname{Gd}(\mathrm{TTFA})_{3}$ solution, which also occurs at 3,000 $\mu \mathrm{Eqv} / \mathrm{L}$, and are over 4 times greater than the maximum values for the HTTFA (22.0), NaTTFA (16.0), and Na+HTTFA one-to-one solution (26.0), which all occurred at the highest concentration, $30,000 \mu \mathrm{Eqv} / \mathrm{L}$.

The results reported above show that increasing the concentration of the TTFA anions or HTTFA molecules results in an increase of the EuMac-Strept. luminescence, until saturation is reached. Similarly, the addition of Gd(III) increases the Eu-Mac-Strept. luminescence, and eventually results in saturation. The presence of Gd(III) can also change the concentration of the TTFA anion and/or HTTFA molecule at which maximum luminescence occurs. Since at these concentrations of NaTTFA the ligand binding sites of the EuMac are fully saturated by TTFA ligands, the excess TTFA anions must be a significant source of the energy emitted by the EuMac. Unexpectedly, as shown in Table 2, the relative net luminescence of the one-component solutions with molecular HTTFA is approximately equal to that of the one-component solutions with NaTTFA. Thus, in this case the excess HTTFA transfers energy to the EuMac by a means other than homogeneous resonance energy transfer. Since the preponderance of HTTFA is unionized and the acceptor is the TTFA anion, the species are different. Thus, the energy transfer is heterogeneous. In this regard, the HTTFA is behaving like a standard fluorophore or lumiphore, or both. This finding opens the possibility of new means for energy transfer to LnMacs and other energy transfer acceptor lanthanide(III) complexes, utilizing as energy transfer donors conventional, including commercially available, fluorophores or lumiphores.

\subsection{EuMac and TbMac Studies}

The luminescence of two unfunctionalized Quantum Dyes, the EuMac-un and the TbMac-un, was measured in the presence of each of two energy transfer species, $\mathrm{Na}_{2} \mathrm{PDCA}$ and $\mathrm{Na}_{3} \mathrm{Gd}(\mathrm{PDCA})_{3}$, as described for the second set of studies outlined in Section 2.4. In these experiments, all wells contained the same quantity of EuMac-un (1.26 nmol) or TbMacun $(1.29 \mathrm{nmol})$. In the $\mathrm{Na}_{2} \mathrm{PDCA}$ studies, the quantity of $\mathrm{Na}_{2} \mathrm{PDCA}$ increased in the order $0.0 \mathrm{nmol}, 6.3 \mathrm{nmol}, 12.5 \mathrm{nmol}$, $62.6 \mathrm{nmol}, 125 \mathrm{nmol}$, and $188 \mathrm{nmol}$. For $\mathrm{Na}_{2} \mathrm{PDCA}$, the quantity of material expressed as moles of $\mathrm{Na}_{2} \mathrm{PDCA}$ is equal to that expressed as equivalents (Eqv.) of PDCA anion. In the $\mathrm{Na}_{3} \mathrm{Gd}(\mathrm{PDCA})_{3}$ studies, the quantity of PDCA anionic ligand present in the $\mathrm{Na}_{3} \mathrm{Gd}(\mathrm{PDCA})_{3}$ complex increased in the order $0.00 \mathrm{nEqv}, 26 \mathrm{nEqv}, 130 \mathrm{nEqv}, 260 \mathrm{nEqv}, 390 \mathrm{nEqv}$, and $520 \mathrm{nEqv}$. The solutions were allowed to dry by storing the plates for $24 \mathrm{hr}$. at room temperature and in the dark. Four sets of data were measured: EuMac-un with $\mathrm{Na}_{2} \mathrm{PDCA}$ and with $\mathrm{Na}_{3} \mathrm{Gd}(\mathrm{PDCA})_{3}$, and TbMac-un with $\mathrm{Na}_{2} \mathrm{PDCA}$ and with $\mathrm{Na}_{3} \mathrm{Gd}(\mathrm{PDCA})_{3}$. 

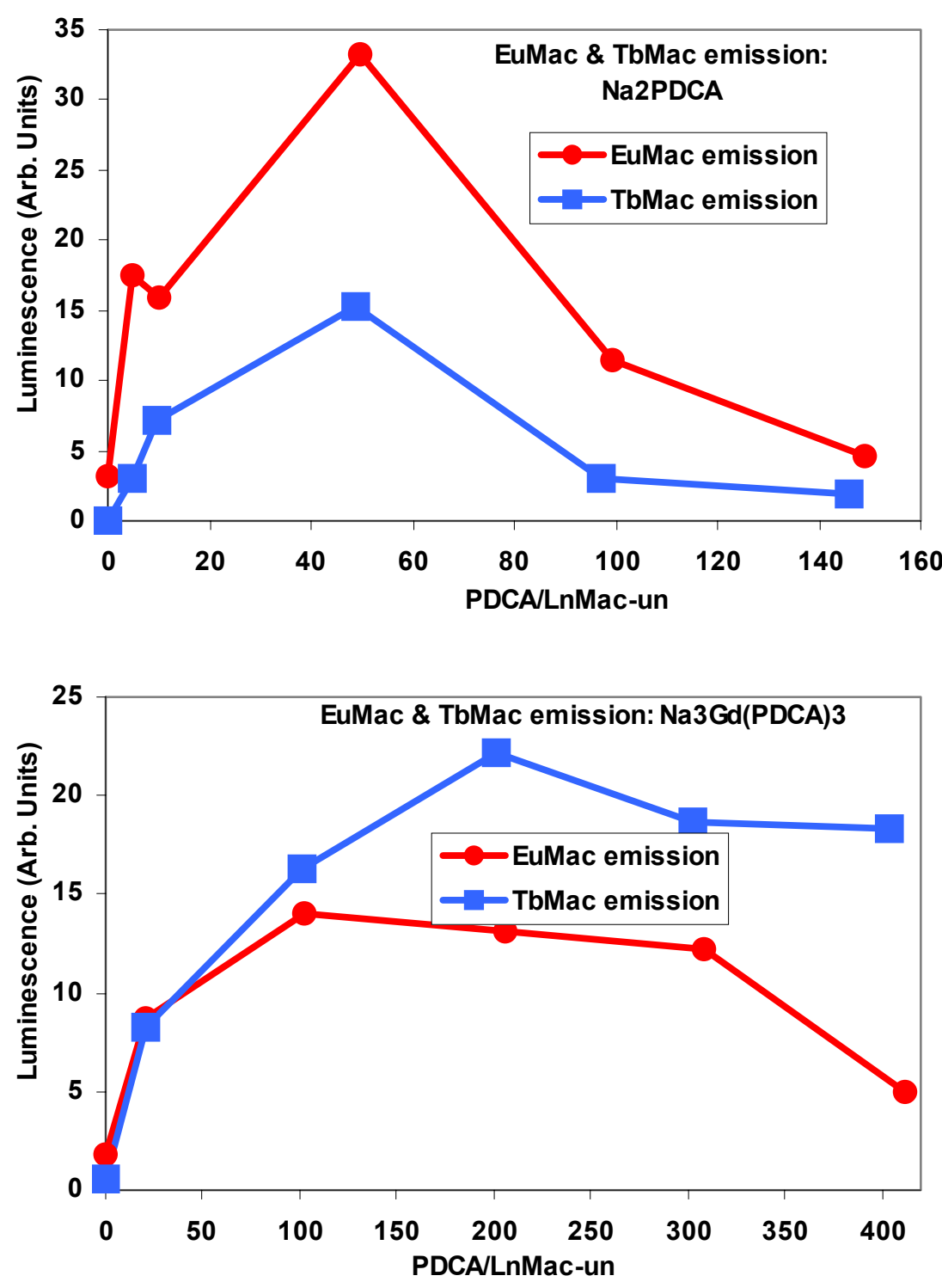

Figure 5a shows the effect of different concentrations of $\mathrm{Na}_{2}$ PDCA on the luminescence of two lanthanide macrocycles, EuMac-un and TbMac-un.
Figure $5 \mathrm{~b}$ shows the effect of different concentrations of $\mathrm{Na}_{3} \mathrm{Gd}(\mathrm{PDCA})_{3}$ on the luminescence of two lanthanide macrocycles, EuMac-un and TbMac-un.

The emission intensity data from 7 individual repeats of the dried methanolic solutions were averaged. In Figure 5a and Figure 5b, the luminescence data for the EuMac-un are reported as the average of the values between 612 and $621 \mathrm{~nm}$, and the data for the TbMac-un are reported as the average of the values between 540 and $550 \mathrm{~nm}$. The ordinates (luminescence) of Figures $5 \mathrm{a}$ and $5 \mathrm{~b}$ are in the same arbitrary units. The abscissas of Figures $5 \mathrm{a}$ and $5 \mathrm{~b}$ are the ratio between the equivalents of PDCA anions, which is the same as the moles of the $\mathrm{Na}_{2} \mathrm{PDCA}$, and the moles of the lanthanide macrocycles. The presence of an excess of the PDCA anion significantly increases the luminescence of the EuMac-un and TbMacun after both are already saturated by PDCA. The simplest explanation for this effect is that the excess PDCA anions, while not bound to the LnMac-un, can excite by homogeneous resonance energy transfer the LnMac-bound PDCA anions, which then transfer energy to the emitting lanthanide. Another possible explanation is that the unbound PDCA anions can transfer energy directly to the lanthanide ions of the LnMac-un complexes.

In contrast to the results obtained in the studies with TTFA (Table 1), the inclusion of Gd(III) decreases the luminescence enhancing effect of the PDCA anions for the EuMac-un (Figure 5b). However, for the TbMac-un the inclusion of Gd(III) 
from the $\mathrm{Na}_{3} \mathrm{Gd}(\mathrm{PDCA})_{3}$ (Figure $5 \mathrm{~b}$ ) increases the luminescence as well as the concentration of the anion required to maximize luminescence. Thus, in samples containing PDCA the replacement of the sodium cation by the gadolinium cation can selectively modulate the relative luminescence of lanthanide macrocycles. This effect may be related to the Gd(III) ion modulating the energy or other property of the electronic levels in the excited PDCA anion, prior to the transfer of energy to the acceptor.

\subsection{Increase in Luminescence During Drying}

Time-gated excitation from a UV LED was employed to study the effect of drying on luminescence intensity. One $\mu \mathrm{L}$ of the $1 \mathrm{mg} / \mathrm{mL}$ EuMac-Strept solution was applied to the fiberglass disk of a chamber slide and allowed to dry. The slide chamber was formed by pressing the coverslip onto the double stick tape previously attached to the slide. The Gd(TTFA) 3 was then applied slowly to the exposed edge of the fiberglass disk, which was allowed to dry slowly. Three hundred $2 \mathrm{~ms}$ images were summed for a total excitation time of $300 \mathrm{~ms}(300 \times 1 \mathrm{~ms})$. A background image was obtained under the same conditions with the light blocked before it entered the camera. The summed images were corrected by subtraction of the background image. Each of the relative luminescence values shown in Figure 6 is the sum of the pixels in the corrected image divided by one million.

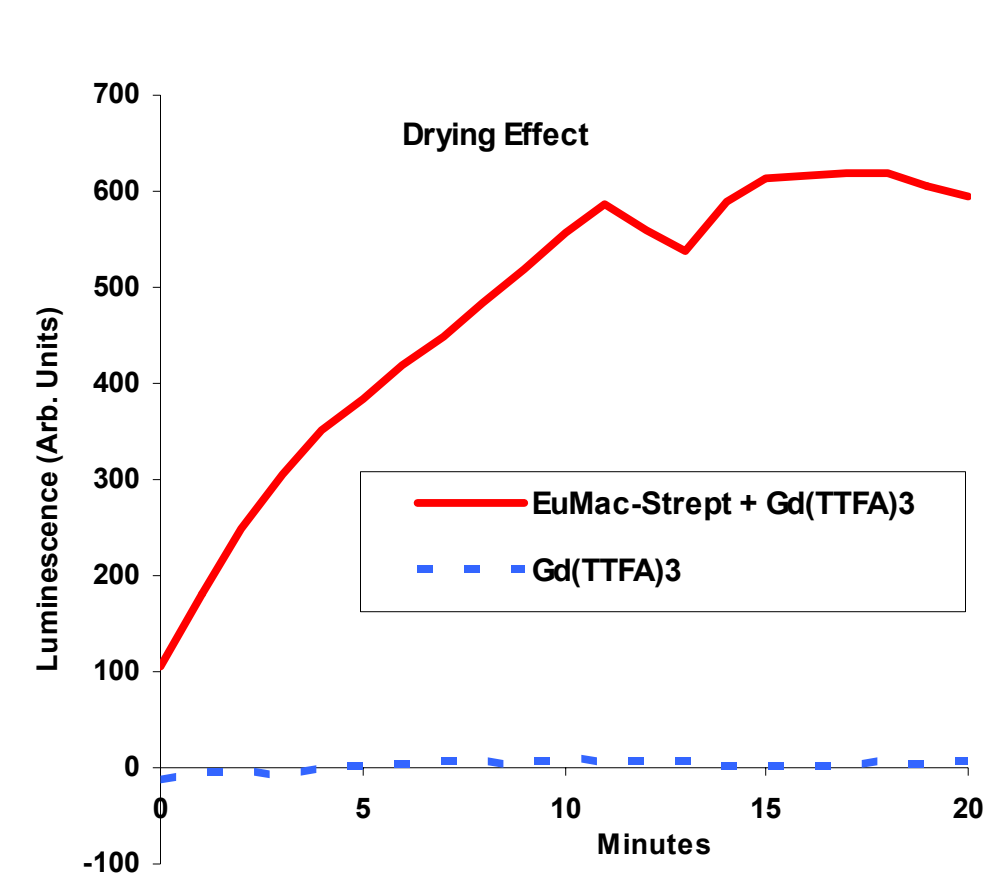

Figure 6. shows the increase in emission caused by drying an ethanolic solution of EuMacStrept $+\mathrm{Gd}(\mathrm{TTFA})_{3}$, and the minimal background emission of Gd(TTFA) $)_{3}$. The excitation period was $1 \mathrm{~ms}$. Each data point is the sum of three hundred $2 \mathrm{~ms}$ images. Both sets of emissions have been corrected for the camera background.

As is shown in Figure 6, the luminescence increases approximately 6 fold during the drying process. Since with the present instrumentation there was a short lag before the zero time measurement was performed, the 6 fold increase is probably an underestimate of the total increase. The values recorded from 12 to 20 minutes are consistent with the sample been dry at 11 minutes. The background due to the $\operatorname{Gd}(\mathrm{TTFA})_{3}$ is minimal. None of the subtractions to produce the corrected images of the EuMac-Strept $+\mathrm{Gd}(\mathrm{TTFA})_{3}$ disks had any negative pixels. However, all subtractions used to produce the corrected images of the control Gd(TTFA) $)_{3}$ disks had a significant number of negative pixels, and for 8 of these more than half of the subtractions resulted in negative values.

\section{Discussion}

Luminescence enhancement of the europium and terbium Quantum Dyes has been achieved with NaTTFA, HTTFA, and $\mathrm{Na}_{2} \mathrm{PDCA}$, eliminating the requirement for a non-luminescent lanthanide ion, such as $\mathrm{Gd}(\mathrm{III})$, to increase the luminescence. In fact, for two of the systems studied here the presence of the Gd(III) ion actually decreased the luminescence. The data summarized in Figure 3 show that the addition of NaTTFA to Gd(TTFA) $)_{3}$ resulted in an increase in lumines- 
cence compared to the pure Gd(TTFA) $)_{3}$. In Figure 4, the data show that the admixture of Gd(TTFA) 3 with NaTTFA or HTTFA resulted in an increase in luminescence compared to the pure Gd(TTFA) $)_{3}$. However, in Figure $5 \mathrm{a}$ and $\mathrm{b}$, where the anion was PDCA, the data showed that the presence of Gd(III) decreased the emission of the EuMac-un relative to that of the TbMac-un. Thus, the model that considers the presence of a second non-luminescent lanthanide ion to promote the formation of the excited triplet state of the energy transfer species ${ }^{14}$, which then transfers an energy quantum to the complexed lanthanide ion emitter, is not sufficient to explain the results reported here.

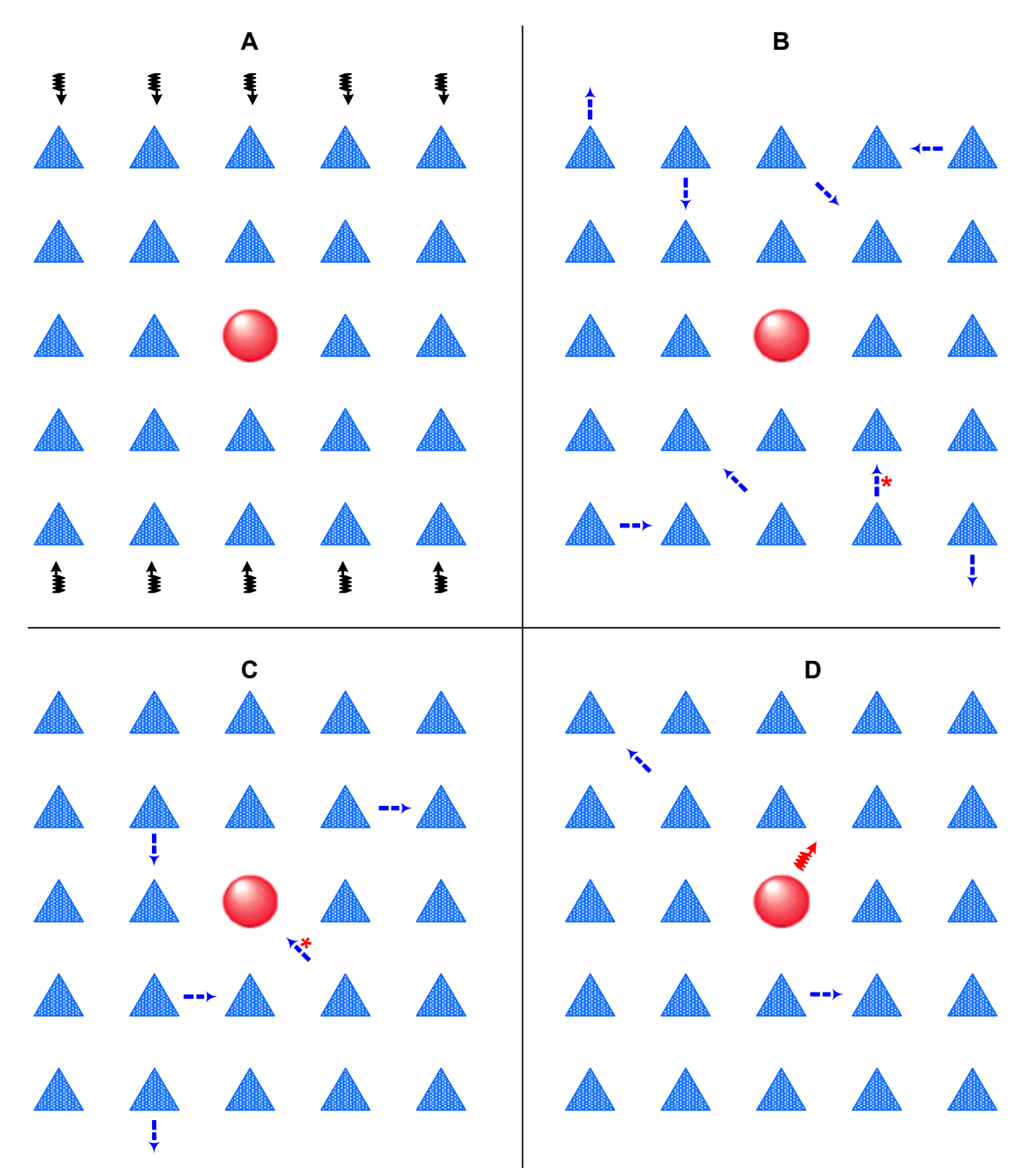

Figure 7. Hypothetical description of the mechanism of FRETEL. In all 4 cartoons (A,B,C,D), the central sphere represents a europium(III) macrocyle with bound ligands. Each of the triangles represents an energy acceptor-donor, which can be an anionic ligand complexed Gd(III), or a free anion, or a HTTFA. molecule.

The heavy squiggly arrow, $m \boldsymbol{m} \rightarrow$, represents a UV excitation photon. The dashed arrow, -->, represents energy that is transferred between acceptor-donors or from an acceptor-donor to the europium macrocycle or one of its ligands. The squigglyarrow, $m \rightarrow$, emanating from the EuMac represents the emission of a red photon.

Figure 7 is a diagrammatic representation of this hypothetical explanation of the FRETEL effect. Figure 7A shows UV photons exciting acceptor-donors. Figure 7B shows the acceptor-donors transferring energy between themselves. Since this energy transfer between acceptor-donors is omnidirectional, most of the quanta will not go in the direction of the relatively rare EuMac. In this example, only the quantum with an asterisk is being transferred to another acceptor-donor that, 
as shown in Figure 7C, can then transfer energy to the EuMac or one of its ligands. In Figure 7D, the EuMac is shown emitting a red photon.

Since the FRETEL effect occurs in the dry state, most of the energy transfer donors present in the system can not be bound directly to the $\mathrm{Eu}(\mathrm{III})$ or $\mathrm{Tb}(\mathrm{III})$ ions of the macrocyclic complex. Thus, the unbound energy transfer donors may transfer energy directly to the central ion, or they may transfer energy by FRET to the coordinated ligands. One possibility is that the mechanism involves homogeneous FRET, with the unbound energy transfer donors exchanging energy quanta among themselves. The quanta transferred between the acceptor-donors have a lower range of energies than the exciting photons shown in Figure 7A, and one of these quanta could eventually excite one of the ligands of the EuMac with the ultimate release of a red photon from the europium ion. Direct excitation of the europium ion could also occur. Menzel et al. ${ }^{15}$ have stated that it is even possible for CdS nanoparticles to sensitize directly the europium ion of a Eu(TTFA) ${ }_{3}$ complex. Since singlet energy transfer has been implicated in the excitation of $\mathrm{Nd}(\mathrm{III})$ complexes ${ }^{16}$, and both the TTFA and the PDCA anions, as well as the unionized HTTFA, can ultimately transfer energy to lanthanide ions in the solid state, it is possible that even species with excited singlet states are capable of increasing the luminescence of lanthanide complexes by fluorescence energy transfer.

\section{Conclusions}

A pair of efficient, chemically identical, narrow band emitting, time-gatable labels, europium and terbium Quantum Dyes, have been developed. This pair of labels now provides the potential for sensitive and convenient ratiometric measurements. The luminescence efficiency of cytological and other solid-phase preparations of the europium and terbium macrocycles, Quantum Dyes, was enhanced by the presence of complexes of non-luminescent lanthanide ions with certain ligands or, in the absence of the non-luminescent lanthanide ions, by an excess of the ligands or other related species. This enhancement, resulting from the FRETEL effect, occurred in thin films prepared by spontaneous evaporation of a solution containing the energy transfer anionic ligands, TTFA or PDCA, and the protonated form of one of the anions, HTTFA. The enhancement occurred both in the presence and in the absence of the gadolinium(III) complex of these ligands. Ethanol, methanol or other volatile organic liquids, as well as water, can be used as solvents. The emission enhancement of the Quantum Dyes by excess ligands in the absence of the Gd(III) ion eliminates the problem of the background emission resulting from trace $\mathrm{Eu}(\mathrm{III})$ contaminant in the Gd(III).

In the case of time-gated measurements and imaging, the use of an UV LED for excitation has the benefit that the excitation time can be extended from the short pulse, ca. 2 microseconds, of the previously used flashlamp ${ }^{11}$, to 1 millisecond or more. This would result in a fifty thousand fold increase in the permissible excitation period of a Quantum Dye compared to that of a quantum dot (20 nanoseconds) or conventional fluorophore, compensating for the much smaller molar extinction coefficients of the Quantum Dyes. This extended excitation period can be combined with the FRETEL effect, and with the capacity--owing to the lack of concentration quenching--to load peptide carriers with multiple Quantum Dyes ${ }^{17,18}$. The long excitation period also allows the use of high resolution, relatively inexpensive CCD cameras instead of image-intensified cameras. Thus, a simple and relatively inexpensive protocol is now available for taking advantage of the Quantum Dyes' narrow emissions and their capacity for time-gating.

\section{ACKNOWLEDGEMENTS}

This project was supported by Newport Instruments internal development funds, by Phoenix Flow Systems internal development funds, and by the Vallarino research fund of Virginia Commonwealth University. The comments, encouragement, and suggestions of C. Kevin Becker, Stephanie H. Leif, and Suzanne B. Leif are gratefully appreciated.

\section{REFERENCES}

1. J. R. Lakowicz, Principles of Fluorescence Spectroscopy, Second Edition, p.87, Kluwer Academic/Plenum Publishers, New York, 1999.

2. S. I. Weissman, "Intramolecular Energy Transfer: The Fluorescence of Complexes of Europium”, The Journal of Chemical Physics, 10, 214-217, 1942.

3. Y.Y. Xu and I. A. Hemmila, "Co-fluorescence Enhancement System Based on Pivaloyltrifluoroacetone and Yttrium for the Simultaneous Detection of Europium, Terbium, Samarium and Dysprosium”, Analytica Chimica Acta, 256, 9-16, 1992.

4. R. B. Mujumdar, L. A. Ernst, S. R. Mujumdar, C. J. Lewis, and A. S. Waggoner, "Cyanine Dye Labeling Reagents: Sulfoindocyanine Succinimidyl Esters", Bioconjug Chem, 4, 105-111, 1993. 
5. R. C. Leif. and L. M. Vallarino, “A Reagent System and Method for Increasing the Luminescence of Lanthanide(III) Macrocyclic Complexes”, US Patent 6,340,744, 2002.

6. R. C. Leif. and L. M. Vallarino, “A Reagent System and Method for Increasing the Luminescence of Lanthanide(III) Macrocyclic Complexes”, US Patent 6,750,005, 2004.

7. A. J. Bromm Jr., R. C. Leif, J. R. Quagliano, and L. M. Vallarino, “The Addition of a Second Lanthanide Ion to Increase the Luminescence of Europium(III) Macrocyclic Complexes", Proceedings of Optical Diagnostics of Living Cells II, D. L. Farkas, R. C. Leif, B. J. Tromberg, Editors, 3604, 263-272, SPIE, Bellingham, WA. 1999.

8. C. Tong, Y. Zhub, and W. Liua, 'Study on the Co-luminescence System of Dy-Gd-1,6-bis(1'phenyl-3'-methyl-5'-pyrazol-4'-one)hexanedione-cetyltrimethylammonium Bromide and Its Analytical Application", Analyst, 126, 1168-1171, 2001.

9. R. C. Leif, M. C. Becker, A. Bromm Jr., N. Chen, A. E. Cowan, L. M. Vallarino, S. Yang, and R. M. Zucker, "Lanthanide Enhanced Luminescence (LEL) with One and Two Photon Excitation of Quantum Dyes ${ }^{\circledR}$ Lanthanide(III)-Macrocycles", Manipulation and Analysis of Biomolecules, Cells, and Tissues, D. V. Nicolau, J. Enderlein, R. C. Leif, and D. Farkas, Editors, 5322, 187-199, SPIE, Bellingham, WA. 2004.

10. R. C. Leif and L. M. Vallarino, “A Reagent System and Method for Increasing the Luminescence of Lanthanide(Iii) Macrocyclic Complexes", (Patent application filed Nov. 2004).

11. R.C. Leif, M.C. Becker, L.M. Vallarino J.W. Williams, and S. Yang, "Progress in the Use of Quantum Dye ${ }^{\circledR}$ Eu(III)-Macrocycles", Manipulation and Analysis of Biomolecules, Cells and Tissues, D. V. Nicolau, J. Enderlein, and R. C. Leif, Editors, 4962, 341-353, SPIE, Bellingham, WA. 2003.

12. A. J. Bromm Jr., R. C. Leif, J. R. Quagliano, and L. M. Vallarino, "The Addition of a Second Lanthanide Ion to Increase the Luminescence of Europium(III) Macrocyclic Complexes", Proceedings of Optical Diagnostics of Living Cells II, D. L. Farkas, R. C. Leif, B. J. Tromberg, 3604, 263-272, SPIE, Bellingham, WA. 1999.

13. A. Kawski, "Excitation Energy Transfer and Its Manifestation in Isotropic Media", Photochem Photobiol, 38, 487, 1983.

14. Y. Yang and S. Zhang, "Spectral Study of the Co-luminescence Effect of Lanthanide Ternary Complexes with Benzoic Acid and Phenanthroline", Spectroscopy Letters, 37, 1-10, 2004.

15. E. R. Menzel, K. K Bouldin, R. H. Murdock, Trace Explosives Detection by Photoluminescence, The Scientific World JOURNAL, 4, 55-66, 2004.

16. G. A. Hebbink, S. I. Klink, L. Grave, P. G. B. Oude Alink, and F. C. J. M. van Veggel, "Singlet Energy Transfer as the Main Pathway in the Sensitization of Near-Infrared $\mathrm{Nd}^{3+}$ Luminescence by Dansyl and Lissamine Dyes", Chemphyschem, 3, 1014 -1018, 2002. 17. R. C. Leif. and L. M. Vallarino, "Conjugated Polymer Tag Complexes”, PCT WO 01/27625 A1, 19 April, 2001.

18. R. C. Leif, M. C. Becker, A. J. Bromm Jr., L. M. Vallarino, S. A. Williams, and S. Yang, "Increasing the Luminescence of Lanthanide(III) Macrocyclic Complexes by the Use of Polymers and Lanthanide Enhanced Luminescence", Optical Diagnostics of Living Cells IV, D. L. Farkas and R. C. Leif, 4260, 184-197, SPIE, Bellingham, WA. 2001. 